\title{
7. Conclusions
}

The investigation of radiation-induced interstitial atoms by means of mechanical relaxation techniques has provided valuable basic information about the structural and diffusional properties of such defects. The anisotropy factors $\pi^{(i)}$ provide the elastic dipole moments and the symmetry of the elastic dipole tensor which in turn can be used to test possible atomistic defect models. On the other hand, the relaxation strength has been used to follow the reactions of individual kinds of defects during isochronal annealing treatments. This was possible due to the fact that the relaxation lines of different kinds of defects distinguish themselves by their characteristic temperature locations. Thus, individual defects can be followed separately even in the presence of a larger ensemble of different species.

The variation of the temperature location of individual relaxation lines with the experimentally determined response times, e.g. vibrational period and elastic aftereffect times, yields the activation energy and the preexponential of the underlying diffusion process. In this sense, the case of the single self-interstitial in electron-irradiated Aluminum might be considered as a keyexperiment and a highlight of the whole subject.

On the other hand, examples like electron-irradiated Copper or Molybdenum clearly reveal the limitations of the mechanical relaxation technique in that they show that orientationally undistinguishable defects remain undetected. In such cases - of course also in the former - full advantage can be taken from the complimentary nature of Huang diffuse x-ray scattering measurements. The latter ones provide the structural properties also in the case of isotropic or immobile defects, although they are insensitive to any effect involving the mobility of defects.

The interest in irradiation-induced interstitial atoms in alloys was originally related to phenomena in fission and fusion reactor materials. However, the significance of this subject extends into the more general field of nonequilibrium phenomena as they occur during ion implantation and ion beam processing of materials. 